\title{
TOWARD A CLASSIFICATION OF KILLING VECTOR FIELDS OF CONSTANT LENGTH ON PSEUDO-RIEMANNIAN NORMAL HOMOGENEOUS SPACES
}

\author{
Joseph A. Wolf, Fabio Podestà \& Ming Xu
}

\begin{abstract}
In this paper we develop the basic tools for a classification of Killing vector fields of constant length on pseudo-Riemannian homogeneous spaces. This extends a recent paper of $\mathrm{M}$. $\mathrm{Xu}$ and J. A. Wolf, which classified the pairs $(M, \xi)$ where $M=G / H$ is a Riemannian normal homogeneous space, $G$ is a compact simple Lie group, and $\xi \in \mathfrak{g}$ defines a nonzero Killing vector field of constant length on $M$. The method there was direct computation. Here we make use of the moment map $M \rightarrow \mathfrak{g}^{*}$ and the flag manifold structure of $\operatorname{Ad}(G) \xi$ to give a shorter, more geometric proof which does not require compactness and which is valid in the pseudo-Riemannian setting. In that context we break the classification problem into three parts. The first is easily settled. The second concerns the cases where $\xi$ is elliptic and $G$ is simple (but not necessarily compact); that case is our main result here. The third, which remains open, is a more combinatorial problem involving elements of the first two.
\end{abstract}

\section{Introduction}

We consider a connected real reductive Lie group $G$, a nondegenerate invariant bilinear form $b$ on $\mathfrak{g}$, and a closed reductive subgroup $H$ in $G$ such that $b$ is nondegenerate on $\mathfrak{h}$. Decompose $\mathfrak{g}=\mathfrak{h}+\mathfrak{m}$ where $\mathfrak{m}$ is the $b$-orthocomplement of $\mathfrak{h}$. Then $b$ is nondegenerate on $\mathfrak{m}$ and induces a pseudo-Riemannian metric $d s^{2}$ on $M=G / H$. Those are our normal pseudo-Riemannian metrics. This includes the Riemannian case, where $d s^{2}$ is either positive definite (as usual) or negative definite (so that $b$ can be the Killing form when $G$ is a compact semisimple Lie group).

JAW: Research partially supported by a Simons Foundation grant and by the Dickson Emeriti Professorship at the University of California, Berkeley.

MX: Research supported by NSFC no. 11271216, State Scholarship Fund of CSC (no. 201408120020), Science and Technology Development Fund for Universities and Colleges in Tianjin (no. 20141005), Doctor fund of Tianjin Normal University (no. 52XB1305). Corresponding author.

Received April 6, 2015. 
Note the dependence on the pair $(G, b)$. If $G^{\prime}$ is another transitive group of isometries of $\left(M, d s^{2}\right)$ then $d s^{2}$ need not be normal as a homogeneous space of $G^{\prime}$.

Let $\xi \in \mathfrak{g}$. It induces a Killing vector field on $M$ which we denote $\xi^{M}$. If $x \in M$ then $\xi_{x}^{M}$ is the corresponding tangent vector at $x$. We say that $\xi^{M}$ has constant length (perhaps pseudo-length would be a better term) if the function $x \mapsto d s^{2}\left(\xi_{x}^{M}, \xi_{x}^{M}\right)$ is constant on $M$. The goal of this paper is the classification of triples $(G, H, \xi)$ where $\xi \in \mathfrak{g}$ is nonzero and elliptic, and where $\xi^{M}$ has constant length.

In the setting of pseudo-Riemannian manifolds, constant length Killing vector fields (also called Clifford-Killing or CK vector fields; see $[\mathbf{1 1}]$ ) are the appropriate replacement for isometries of constant displacement (Clifford-Wolf or CW isometries).

In Section 2, we discuss a flag manifold $G_{\mathbb{C}} / Q$ that connects the moment map for conjugation orbits in $\mathfrak{g}$ with the length function for $\xi^{M}$. Then in Section 3, we develop a method of passage through the complex domain that carries this connection to flag domains and the pseudoRiemannian setting. In Section 4, we use these tools to carry out the classification for the cases where $G_{\mathbb{C}}$ is simple; the main result is Theorem 4.4. Those tools don't apply directly to the case where $G$ is simple but $G_{\mathbb{C}}$ is not, but in Section 5 , we use other methods to carry out the classification; there the main result is Theorem 5.2. Section 6 summarizes these classifications to give one of the two main results of this paper, Theorem 6.1. As a consequence of these classifications, Corollary 6.2 indicates the pseudo-Riemannian analog of the correspondence between homogeneity for quotient manifolds and isometries of constant displacement.

The other principal result is Theorem 7.6, which in effect describes current progress toward a classification where $G$ need not be simple.

Let $p r_{\mathfrak{h}}$ and $p r_{\mathfrak{m}}$ denote the respective orthogonal projections of $\mathfrak{g}$ to $\mathfrak{h}$ and $\mathfrak{m}$. Then $d s^{2}\left(\xi_{x}^{M}, \xi_{x}^{M}\right)=b\left(p r_{\mathfrak{m}}(\operatorname{Ad}(g) \xi), p r_{\mathfrak{m}}(\operatorname{Ad}(g) \xi)\right)$ where $x=g H$. Since $b(\operatorname{Ad}(g) \xi, \operatorname{Ad}(g) \xi)$ is independent of $g \in G$, and

$$
\begin{aligned}
& b(\operatorname{Ad}(g) \xi, \operatorname{Ad}(g) \xi)= \\
& \quad b\left(p r_{\mathfrak{h}}(\operatorname{Ad}(g) \xi), p r_{\mathfrak{h}}(\operatorname{Ad}(g) \xi)\right)+b\left(p r_{\mathfrak{m}}(\operatorname{Ad}(g) \xi), p r_{\mathfrak{m}}(\operatorname{Ad}(g) \xi)\right),
\end{aligned}
$$

Lemma 1.1. Let $\xi \in \mathfrak{g}$. Then $\xi^{M}$ has constant length if and only if

$$
f_{\xi}(g):=b\left(p r_{\mathfrak{h}}(\operatorname{Ad}(g) \xi), p r_{\mathfrak{h}}(\operatorname{Ad}(g) \xi)\right)
$$

is independent of $g \in G$.

In view of Lemma 1.1 and our assumption that $G$ is connected, the constant length property for $\xi^{M}$ depends only on the pair $(\mathfrak{g}, \mathfrak{h})$. Thus we can (and will) be casual about passing to and from covering groups of $G$ and about connectivity of $H$. In practice this will be only a matter of whether it is more convenient to write Spin or SO. 


\section{The flag domain}

Recall that $G$ is a real reductive Lie group and $b$ is a nondegenerate $\operatorname{Ad}(G)$-invariant symmetric bilinear form on its Lie algebra $\mathfrak{g}$. We use $b$ to identify adjoint orbits of $G$ on $\mathfrak{g}$ and coadjoint orbits of $G$ on $\mathfrak{g}^{*}$.

Proposition 2.1. Suppose that $\xi \in \mathfrak{g}$ is elliptic, in other words that ad $(\xi)$ is semisimple (diagonalizable over $\mathbb{C}$ ) with pure imaginary eigenvalues. Let $L$ denote the centralizer of $\xi$ in $G$. Then $G_{\mathbb{C}}$ has a parabolic subgroup $Q$ with the properties

- $L$ is the isotropy subgroup of $G$ at the base point $z_{0}=1 Q$ for the action of $G$ (as a subgroup of $G_{\mathbb{C}}$ ) on the complex flag manifold $Z=G_{\mathbb{C}} / Q$,

- $L_{\mathbb{C}}$ is the reductive part of $Q$,

- the orbit $G\left(z_{0}\right) \subset Z$ is open and carries a $G$-invariant pseudoKähler metric, which can be normalized so that

- $\operatorname{Ad}(g) \xi \mapsto g Q$ is a symplectomorphism of $\mathcal{O}_{\xi}:=\operatorname{Ad}(G) \xi$ onto $G\left(z_{0}\right)$ where the symplectic form on $\mathcal{O}_{\xi}$ is the Kostant-Souriau form $\omega(\eta, \zeta)=b(\xi,[\eta, \zeta])$ and the symplectic form on $G\left(z_{0}\right)$ is the imaginary part of the invariant pseudo-Kähler metric.

In particular, $\mathcal{O}_{\xi}$ has a $G$-invariant pseudo-Kähler structure.

Proof. By construction $L$ is reductive. In fact, $\xi$ is contained in a fundamental (maximally compact) Cartan subalgebra $\mathfrak{t}$ of $\mathfrak{g}$, and $\mathfrak{l}_{\mathbb{C}}$ is $\mathfrak{t}_{\mathbb{C}}$ plus all the $\mathfrak{t}_{\mathbb{C} \text {-root spaces }} \mathfrak{g}_{\alpha}$ for roots $\alpha$ that vanish on $\xi$. Define $\mathfrak{q}=\mathfrak{l}_{\mathbb{C}}+\sum_{\alpha(i \xi)<0} \mathfrak{g}_{\alpha}$. It is a parabolic subalgebra of $\mathfrak{g}_{\mathbb{C}}$ with reductive part $\mathfrak{l}_{\mathbb{C}}$.

Let $\tau$ denote complex conjugation of $\mathfrak{g}_{\mathbb{C}}$ over $\mathfrak{g}$. Then $\tau(i \xi)=-i \xi$ so $\mathfrak{q}+\tau \mathfrak{q}=\mathfrak{g}_{\mathbb{C}}$, and also $\mathfrak{q} \cap \tau \mathfrak{q}=\mathfrak{l}_{\mathbb{C}}$. There are two immediate consequences: (i) $G\left(z_{0}\right)$ is open in $Z=G_{\mathbb{C}} / Q$ and (ii) $\operatorname{Ad}(g) \xi \mapsto g Q$ is a diffeomorphism of $\mathcal{O}_{\xi}$ onto $G\left(z_{0}\right)$. Note that (ii) uses simple connectivity of both $Z$ and $\mathcal{O}_{\xi}$.

Since $\mathfrak{q} \cap \tau \mathfrak{q}=\mathfrak{l}_{\mathbb{C}}$, which is reductive, $G\left(z_{0}\right)$ carries a $G$-invariant measure. Any such measure comes from the volume form of an invariant indefinite-Kähler metric; see [9], or see the exposition of flag domains in [2]. This metric is constructed in [9] using an invariant bilinear form; as is the Kostant-Souriau form, and by the construction a proper normalization of the metric has imaginary part equal to the KostantSouriau form.

q.e.d.

Remark 2.2. In our flag domain cases, Proposition 2.1 extends the structural result of $[\mathbf{1}$, Theorem 1.3(4)] from symplectic to pseudoKähler.

Remark 2.3. The parabolic $\mathfrak{q}$ is the sum of the non-positive eigenspaces of ad $(i \xi)$ on $\mathfrak{g}_{\mathbb{C}}$. If $g \in G_{\mathbb{C}}$ now $\operatorname{Ad}(g) \mathfrak{q}$ is the sum of the non-positive eigenspaces of $\operatorname{ad}(\operatorname{Ad}(g) \xi)$ on $\mathfrak{g}_{\mathbb{C}}$. As $Q$ is its own nor- 
malizer in $G_{\mathbb{C}}$ we can identify $Z=G_{\mathbb{C}} / Q$ with the space of $\operatorname{Ad}\left(G_{\mathbb{C}}\right)$ conjugates of $\mathfrak{q}$. Thus, if $S$ is any subgroup of $G_{\mathbb{C}}$, we see exactly how $\operatorname{Ad}(S) \xi \subset Z$.

We are using $b$ to identify $\mathfrak{g}$ with $\mathfrak{g}^{*}$; similarly use $\left.b\right|_{\mathfrak{h}}$ to identify $\mathfrak{h}$ with $\mathfrak{h}^{*}$. The inclusion

$$
\mu_{G}: \mathcal{O}_{\xi} \hookrightarrow \mathfrak{g}
$$

coincides with the moment map for the (necessarily Hamiltonian) action of $G$ on $\mathcal{O}_{\xi}$. Now consider the action of $H$ on $\mathcal{O}_{\xi}$. The corresponding moment map is

$$
\mu_{H}:=p r_{\mathfrak{h}} \circ \mu_{G}: \mathcal{O}_{\xi} \rightarrow \mathfrak{h} .
$$

Thus Lemma 1.1 can be reformulated as

Lemma 2.6. Let $\xi \in \mathfrak{g}$. Then $\xi^{M}$ has constant length if and only if $\zeta \mapsto b\left(\mu_{H}(\zeta), \mu_{H}(\zeta)\right)$ is constant on $\mathcal{O}_{\xi}$.

\section{Holomorphic considerations}

The group $H$ is reductive in $G$ because $b$ is nondegenerate on $\mathfrak{h}$. Thus [4] there is a Cartan involution $\theta$ of $G$ such that $\left.\theta\right|_{H}$ is a Cartan involution on $H$. That gives us the decompositions

$$
\mathfrak{g}=\mathfrak{k}+\mathfrak{p} \text { and } \mathfrak{h}=(\mathfrak{h} \cap \mathfrak{k})+(\mathfrak{h} \cap \mathfrak{p}),
$$

into \pm 1 eigenspaces of $d \theta$.

From now on we suppose that $G$ is semisimple and that $b$ is a positive linear combination of the Killing forms of the simple ideals of $\mathfrak{g}$. Thus $b$ is negative definite on $\mathfrak{k}$ and positive definite on $\mathfrak{p}$. \{The reader can extend many of our results to the case of reductive $G$ by stipulating $b(\mathfrak{k}, \mathfrak{p})=0, b$ negative definite on $\mathfrak{k}$, and $b$ positive definite on $\mathfrak{p}$. $\}$ The decompositions of $\mathfrak{g}$ and $\mathfrak{h}$ give us compact real forms

$$
\begin{aligned}
\mathfrak{g}_{u}=\mathfrak{k}+i \mathfrak{p}=\mathfrak{h}_{u}+\mathfrak{m}_{u}, \\
\qquad \mathfrak{h}_{u}=(\mathfrak{h} \cap \mathfrak{k})+i(\mathfrak{h} \cap \mathfrak{p}) \text { and } \mathfrak{m}_{u}=(\mathfrak{m} \cap \mathfrak{k})+i(\mathfrak{m} \cap \mathfrak{p}),
\end{aligned}
$$

of $\mathfrak{g}_{\mathbb{C}} \mathfrak{h}_{\mathbb{C}}$ and $\mathfrak{m}_{\mathbb{C}}$. Let $G_{u}$ and $H_{u}$ denote the compact real forms of $G_{\mathbb{C}}$ and $H_{\mathbb{C}}$ corresponding to $\mathfrak{g}_{u}$ and $\mathfrak{h}_{u}$.

Extend $b$ to a $\mathbb{C}$-bilinear form $b_{\mathbb{C}}$ on $\mathfrak{g}_{\mathbb{C}}$. Then $b_{u}:=\left.b_{\mathbb{C}}\right|_{\mathfrak{g}_{u}}$ is negative definite. As $b(\mathfrak{h}, \mathfrak{m})=0$ we have $b_{\mathbb{C}}\left(\mathfrak{h}_{\mathbb{C}}, \mathfrak{m}_{\mathbb{C}}\right)=0$ and thus $b_{u}\left(\mathfrak{h}_{u}, \mathfrak{m}_{u}\right)=$ 0 . The orthogonal projection $p r_{\mathfrak{h}_{\mathbb{C}}}: \mathfrak{g}_{\mathbb{C}} \rightarrow \mathfrak{h}_{\mathbb{C}}$ restricts to orthogonal projection $p r_{\mathfrak{h}_{u}}: \mathfrak{g}_{u} \rightarrow \mathfrak{h}_{u}$.

Lemma 3.1. Define $f_{\xi}: G_{\mathbb{C}} \rightarrow \mathbb{C}$ by

$$
f_{\xi}(g)=b_{\mathbb{C}}\left(p r_{\mathfrak{h}_{\mathbb{C}}}(\operatorname{Ad}(g) \xi), p r_{\mathfrak{h}_{\mathbb{C}}}(\operatorname{Ad}(g) \xi)\right) .
$$

Then $f_{\xi}$ is holomorphic.

Proof. The map $g \mapsto \operatorname{Ad}(g) \xi$ is holomorphic on $G_{\mathbb{C}}$, the projection $p r_{\mathfrak{h}_{\mathbb{C}}}: \mathfrak{g}_{\mathbb{C}} \rightarrow \mathfrak{h}_{\mathbb{C}}$ is holomorphic, and $b_{\mathbb{C}}$ is complex bilinear. q.e.d. 
Lemma 3.2. $\xi^{M}$ has constant length if and only if $f_{\xi}: G_{\mathbb{C}} \rightarrow \mathbb{C}$ is constant.

Proof. If $\xi^{M}$ has constant length then $f_{\xi}$ is constant on $G$. Since $G$ is a real form of $G_{\mathbb{C}}$ and $f_{\xi}$ is holomorphic, it follows that $f_{\xi}$ is constant.

q.e.d.

Denote $M_{u}=G_{u} / H_{u}$ where $M_{u}$ carries the normal homogeneous Riemannian metric defined by $\left.b_{u}\right|_{\mathfrak{m}_{u}}$. In effect it is the natural compact real form of the affine algebraic variety $M_{\mathbb{C}}=G_{\mathbb{C}} / H_{\mathbb{C}}$. Extending the notion of Cartan duality from Riemannian symmetric space theory, $M_{u}=G_{u} / H_{u}$ is the compact dual to $M=G / H$. If $\xi \in \mathfrak{k}$, in particular if $\xi \in \mathfrak{g}_{u}$, we write $\xi^{M_{u}}$ for the corresponding vector field on $M_{u}$. Now Lemmas 2.6 and 3.2 give us

Proposition 3.3. If $\xi \in \mathfrak{k}$ and $\xi^{M}$ has constant length on $M$ if, and only if, $\xi^{M_{u}}$ has constant length on the Riemannian normal homogeneous space $M_{u}:=G_{u} / H_{u}$.

\section{Classification for $G_{\mathbb{C}}$ simple}

In this section, we carry out the classification of constant length Killing vector fields $\xi^{M}$, on reductive normal homogeneous pseudoRiemannian manifolds $M=G / H$ when the group $G_{\mathbb{C}}$ is simple. The compact version of this classification was done by direct computation in [11], but here we have a less computational approach that starts with classification ([5], or see [6]) of Onishchik for irreducible complex flag manifolds $Z=G_{u} / L_{u}$, on which a proper closed subgroup $H_{u}$ of $G_{u}$ acts transitively. On the other hand we need the classification where $H$ need not be compact. For that we use methods from [10]. In Section 5, we give a separate argument to deal with the case where $G$ is simple but $G_{\mathbb{C}}$ is not. Then in Section 6, we translate those results to the classification of constant length Killing vector fields $\xi^{M}$ on reductive normal homogeneous pseudo-Riemannian manifolds $M=G / H$, with $G$ simple and $\xi$ nonzero and elliptic.

For clarity of exposition we always assume that $G_{\mathbb{C}}$ is connected and simply connected, that the real forms $G$ and $G_{u}$ are analytic subgroups of $G_{\mathbb{C}}$, and that $H, H_{\mathbb{C}}$ and $H_{u}$ are analytic subgroups of $G, G_{\mathbb{C}}$ and $G_{u}$.

Proposition $4.1([5])$. Consider a complex flag manifold $Z=G_{\mathbb{C}} / Q$. Suppose that $Z$ is irreducible, i.e., that $G_{\mathbb{C}}$ is simple. Then the closed connected subgroups $H_{u} \subset G_{u}$ transitive on $Z,\{1\} \neq H_{u} \varsubsetneqq G_{u}$, are precisely those given as follows:

1. $Z=S U(2 n) / U(2 n-1)=P^{2 n-1}(\mathbb{C})$, complex projective $(2 n-1)$ space; there $G_{\mathbb{C}}=S L(2 n ; \mathbb{C})$ and $H_{u}=S p(n)$.

2. $Z=S O(2 n+2) / U(n+1)$, unitary structures on $\mathbb{R}^{2 n+2}$; there $G_{\mathbb{C}}=S O(2 n+2 ; \mathbb{C})$ and $H_{u}=S O(2 n+1)$. 
3. $Z=\operatorname{Spin}(7) /(\operatorname{Spin}(5) \cdot \operatorname{Spin}(2))$, nonsingular complex quadric; there $G_{\mathbb{C}}=\operatorname{Spin}(7 ; \mathbb{C})$ and $H_{u}$ is the compact exceptional group $G_{2}$.

Here is the noncompact version of Proposition 4.1.

Proposition 4.2. Consider a complex flag manifold $Z=G_{\mathbb{C}} / Q$ with $G_{\mathbb{C}}$ simple. Here is a complete list of the connected subgroups $H \subset G$ with $H \neq\{1\}$ and $H_{u}$ transitive on $Z$.

1. $Z=S U(2 n) / U(2 n-1)=P^{2 n-1}(\mathbb{C})$ and $H_{u}=S p(n)$. Then $(G, H)$ is one of

(i) $(S U(2 p, 2 q), S p(p, q))$ with $p+q=n$ or

(ii) $(S L(2 n ; \mathbb{R}), S p(n ; \mathbb{R}))$.

2. $Z=S O(2 n+2) / U(n+1)$ and $H_{u}=S O(2 n+1)$. Then $(G, H)$ must be

(i) $(S O(2 p+1,2 q+1), S O(2 p+1,2 q))$ with $p+q=n$ or

(ii) $(S O(2 p+2,2 q), S O(2 p+1,2 q))$ with $p+q=n$.

3. $Z=\operatorname{Spin}(7) /(\operatorname{Spin}(5) \cdot \operatorname{Spin}(2))$ and $H_{u}=G_{2}$. Then the pair $(G, H)$ must be

(i) $\left(\operatorname{Spin}(7), G_{2}\right)$ or

(ii) $\left(\operatorname{Spin}(3,4),\left(G_{2}\right)_{\mathbb{R}}\right)$. (Here $\left(G_{2}\right)_{\mathbb{R}}$ is the split real form of $\left.\left(G_{2}\right)_{\mathbb{C}}\right)$.

Proof. Suppose $Z=S U(2 n) / U(2 n-1 ; \mathbb{C})=P^{2 n-1}(\mathbb{C})$ and $H_{u}=$ $S p(n)$. The real forms of $\left(H_{u}\right) \mathbb{C}=S p(n ; \mathbb{C})$ are the $S p(p, q), p+q=n$, and $S p(n ; \mathbb{R})$, and the real forms of $\left(G_{u}\right)_{\mathbb{C}}=S L(2 n ; \mathbb{C})$ are the $S U(r, s)$, $r+s=2 n$ and the special linear groups $S L(2 n ; \mathbb{R})$ and $S L(n ; \mathbb{H})$.

If $G=S U(r, s)$ and $J=\left(\begin{array}{cc}I_{r} & 0 \\ 0 & -I_{s}\end{array}\right)$ then $G=\left\{g \in S L(2 n ; \mathbb{C}) \mid g \cdot J \cdot{ }^{t} \bar{g}=J\right\}$. Thus $H \neq S p(n ; \mathbb{R})$, for that group cannot have both a symmetric and an antisymmetric bilinear invariant on $\mathbb{R}^{2 n}$. Now $G=S U(r, s)$ implies $H=S p(p, q)$, which in turn implies $G=S U(2 p, 2 q)$. Also, if $G=S L(2 n ; \mathbb{R})$ then $H \approx S p(p, q)$ so $H=S p(n ; \mathbb{R})$.

Next suppose $Z=S O(2 n+2) / U(n+1)$ and $H_{u}=S O(2 n+1)$. The real forms of $\left(H_{u}\right)_{\mathbb{C}}=S O(2 n+1 ; \mathbb{C})$ are the $S O(r, s)$ with $r+s=2 n+1$, and the real forms of $\left(G_{u}\right)_{\mathbb{C}}=S O(2 n+2 ; \mathbb{C})$ are the $S O(k, \ell)$ with $k+\ell=2 n+2$ and $S O^{*}(2 n+2)$. The maximal compact subgroup of $S O^{*}(2 n+2)$ is $U(n+1)$, which does not contain any $S O(r) \times S O(s)$ with $r+s=2 n+1$; so $G \neq S O^{*}(2 n+2)$. Thus $G=S O(k, \ell)$ and $H=S O(r, s)$ with $r \leqq k, s \leqq \ell$ and $k+\ell=r+s+1$, as asserted.

Finally, suppose $Z=\operatorname{Spin}(7) /(\operatorname{Spin}(5) \cdot \operatorname{Spin}(2))$ and $H_{u}=G_{2}$. The real forms of $\left(G_{u}\right)_{\mathbb{C}}=\operatorname{Spin}(7 ; \mathbb{C})$ are the $\operatorname{Spin}(a, b)$ with $a+b=7$, and the real forms of $\left(H_{u}\right)_{\mathbb{C}}=\left(G_{2}\right)_{\mathbb{C}}$ are the compact form $G_{2}$ and the split form $\left(G_{2}\right)_{\mathbb{R}}$. Now $[\mathbf{8}$, Theorem 3.1] completes the argument that $G / H$ is $\operatorname{Spin}(7) / G_{2}$ or $\operatorname{Spin}(3,4) /\left(G_{2}\right) \mathbb{R}$. $\quad$ q.e.d.

To continue we need

Theorem 4.3 (Gori \& Podestà [3]). Suppose $M$ is a compact Kähler $K$-Hamiltonian manifold, where $K$ is a compact connected Lie group 
acting effectively and isometrically on $M$. If $\mu$ denotes the moment map, then the squared moment map $f=|\mu|^{2}$ is constant if and only if $K$ is semisimple and the manifold $M$ is biholomorphically and $K$ equivariantly isometric to the product of a flag manifold and a compact Kähler manifold which is acted on trivially by $K$.

Now we summarize, including the case where $H_{u}$ acts trivially on $Z$. In the proof, one case is eliminated by the requirement that $\xi \in \mathfrak{g}$. The main ingredients in the proof of Theorem 4.4 are Proposition 4.2 and the theorem of Gori and Podestà just quoted.

Theorem 4.4. Suppose that $G$ is absolutely simple, i.e., that $G_{\mathbb{C}}$ is simple. Then there is a nonzero elliptic element $\xi \in \mathfrak{g}$ such that the Killing vector field $\xi^{M}$ on the normal homogeneous space $M=G / H$ has constant length, if and only if, up to finite covering, $(G, H)$ is one of the following pairs:

1. $Z=S U(2 n) / U(2 n-1)=P^{2 n-1}(\mathbb{C})$ and $H_{u}=S p(n)$. Then $(G, H)$ is one of the

$(S U(2 p, 2 q), S p(p, q))$ with $p+q=n$, or is $(S L(2 n ; \mathbb{R}), S p(n ; \mathbb{R}))$.

2. $Z=S O(2 n) / U(n)$ and $H_{u}=S O(2 n-1)$. Then $(G, H)$ is one of the $(S O(2 p, 2 q), S O(2 p-1,2 q))$ with $p+q=n$.

3. $Z=\operatorname{Spin}(7) /(\operatorname{Spin}(5) \cdot \operatorname{Spin}(2))$ and $H_{u}=G_{2}$. Then $(G, H)$ is $\left(\operatorname{Spin}(7), G_{2}\right)$ or $\left(\operatorname{Spin}(3,4),\left(G_{2}\right)_{\mathbb{R}}\right)$.

4. $\mathfrak{h}=0$ and $(G, H)$ is the group manifold pair $(G,\{1\})$.

Proof. Retain the notation of Section 3. We can suppose $\xi \in \mathfrak{k} \subset \mathfrak{g}_{u}$. By Proposition 3.3, $\xi$ induces a Killing vector field $\xi^{M_{u}}$ of constant length on the normal homogeneous Riemannian manifold $M_{u}=G_{u} / H_{u}$. The adjoint orbit $Z:=\operatorname{Ad}\left(G_{u}\right) \xi \subset \mathfrak{g}_{u}$ is endowed with the $G_{u}$-invariant symplectic structure given by the Kostant-Souriau form. The $b$-orthogonal projection $p r_{\mathfrak{h}}: Z \rightarrow \mathfrak{h}_{u}$ defines a moment map $\mu$ for the Hamiltonian action of $H_{u}$ on $Z$. By hypothesis $\mu$ has constant length with respect to $\left.b\right|_{\mathfrak{h}_{u}}$, and by $[\mathbf{3}]$ the flag manifold $Z$ is a Kähler product $Z_{1} \times Z_{2}$ with $H_{u}$ acting transitively on $Z_{1}$ and trivially on $Z_{2}$. Since $G_{\mathbb{C}}$ is simple, either $Z=Z_{1}$ or $Z=Z_{2}$, and if $H_{u}$ is not trivial then $H_{u}$ acts transitively on $Z$. We have shown that $(G, H)$ either is a group manifold or is one of the pairs listed in Propositions 4.1 and 4.2.

In the cases listed in Proposition 4.1, i.e., the cases where $G$ is compact, we already have nonzero elliptic elements $\xi \in \mathfrak{g}$ such that the centralizer of $\xi$ in $G$ is transitive on $G / H$. For $G / H=S U(2 n) / S p(n)$ we use $\xi_{1}=\sqrt{-1} \operatorname{diag}\left\{-(2 n-1), I_{2 n-1}\right\}$; it has centralizer $U(2 n-1)$ in $G$. For $G / H=S O(2 n) / S O(2 n-1)$ we use $\xi_{2}=\operatorname{diag}\{J, \ldots, J\}$ where $J=\left(\begin{array}{cc}0 & 1 \\ -1 & 0\end{array}\right)$; it has centralizer $U(n)$ in $G$. For $G / H=\operatorname{Spin}(7) / G_{2}$ we consider $\mathfrak{s p i n}(5) \oplus \mathfrak{s p i n}(2) \subset \mathfrak{g}$ and take $0 \neq \xi_{3} \in \mathfrak{s p i n}(2)$.

Now consider the noncompact cases listed in Proposition 4.2. Going case by case, $\mathfrak{g}$ contains an appropriate multiple of the $\xi_{i} \in \mathfrak{g}_{u}$ of 
the previous paragraph, with the exception of the homogeneous spaces $S O(2 p+1,2 q+1) / S O(2 p+1,2 q)$. That completes the proof. q.e.d.

Remark 4.5. In the case $(G, H)=(G,\{1\}), M$ is the group manifold, the metric is any nonzero multiple of the Killing form, $G$ acts on itself by left translation, and $\xi$ can be any element of $\mathfrak{g}$ because it is centralized by all right translations. In this case $\xi^{M}$ is of constant length without the requirement that $\xi$ be elliptic.

\section{Classification for $G$ complex simple}

We now look at the case where $G$ is simple but $G_{\mathbb{C}}$ is not. That is when $G$ is the underlying real structure of a complex simple Lie group $E$; then $G_{\mathbb{C}}=E \times \bar{E}$ where $\bar{E}$ is the complex conjugate of $E$ and $G \hookrightarrow G_{\mathbb{C}}$ is the diagonal $\delta E \hookrightarrow G_{\mathbb{C}}$. It is convenient to use the following very general lemma, which is based on the infinitesimal version of $[\mathbf{7}$, Théorème 1]:

Lemma 5.1. Let $\left(M, d s^{2}\right)$ be any connected pseudo-Riemannian homogeneous space. Let $\xi \in \mathfrak{g}$. If the centralizer

$$
L:=\left\{g \in I\left(M, d s^{2}\right) \mid \operatorname{Ad}(g) \xi=\xi\right\}
$$

of $\xi$ in the isometry group $I\left(M, d s^{2}\right)$ has an open orbit on $M$ then $\xi^{M}$ has constant length on $M$. In particular, if $L$ is transitive on $M$ then $\xi^{M}$ has constant length on $M$.

Proof. Let $\mathcal{O}$ be an open $L$-orbit on $M$. If $x, y \in \mathcal{O}$, say $g x=y$ with $g \in L$, then $d s^{2}\left(\xi_{y}^{M}, \xi_{y}^{M}\right)=d s^{2}\left(d g\left(\xi_{x}^{M}\right), d g\left(\xi_{x}^{M}\right)\right)=d s^{2}\left(\xi_{x}^{M}, \xi_{x}^{M}\right)$. Thus $\left\|\xi^{M}\right\|^{2}$ is constant on $\mathcal{O}$. As $\left\|\xi^{M}\right\|^{2}$ is real analytic on $M$ it is constant. q.e.d.

Theorem 5.2. Suppose that $G$ is simple but $G_{\mathbb{C}}$ is not. Then there is a nonzero elliptic element $\xi \in \mathfrak{g}$ such that the Killing vector field $\xi^{M}$ on the normal homogeneous space $M=G / H$ has constant length, if and only if, up to finite covering, $(G, H)$ is one of the pairs
(1) $(S L(2 n ; \mathbb{C}), S p(n ; \mathbb{C}))$,
(2) $(S O(2 n ; \mathbb{C}), S O(2 n-1 ; \mathbb{C}))$,
(3) $\left(\operatorname{Spin}(7 ; \mathbb{C}),\left(G_{2}\right) \mathbb{C}\right)$, or
(4) the group manifold pair $(G,\{1\})$.

Remark 5.3. In all cases of Theorem 5.2, $G / H$ is a complex affine algebraic variety. Also, in the case $(G, H)=(G,\{1\}), M$ is the group manifold, the metric is any nonzero multiple of the Killing form, $G$ acts on itself by left translation, and $\xi$ can be any element of $\mathfrak{g}$ because it is centralized by all right translations. In this case $\xi^{M}$ is of constant length without the requirement that $\xi$ be elliptic. 
Proof. Let $\xi \in \mathfrak{g}$ be nonzero and elliptic. We may assume that it is contained in the Lie algebra $\mathfrak{k}$ of a maximal compact subgroup $K$ of $G$. The point is that it is contained in a fundamental (maximally compact) Cartan subalgebra of $\mathfrak{g}$. All such Cartan subalgebras are $\operatorname{Ad}(G)$ conjugate, and the $\mathfrak{g}$-centralizer of any Cartan subalgebra of $\mathfrak{k}$ is one of them. Thus, for the proof, we may assume $\xi \in \mathfrak{k}$.

Note that $K$ is a compact real form when $G$ is regarded as a complex simple group. Passing to a conjugate, $H$ is stable under the complex conjugation $\tau$ of $G$ with fixed point set $K$, for $\tau$ is a Cartan involution. Now $\mathfrak{g}=\mathfrak{k}+\mathfrak{k}$ under $\tau$ and $\mathfrak{h}=\mathfrak{h} \cap \mathfrak{k}+\mathfrak{h} \cap i \mathfrak{k}$. These are orthogonal decompositions relative to the Killing form of $G$, and the invariant bilinear form $b$ is a positive multiple of that Killing form.

Suppose that $\xi^{M}$ has constant length, equivalently that

$$
b\left(\operatorname{pr}_{\mathfrak{h}}(\operatorname{Ad}(g) \xi), \operatorname{pr}_{\mathfrak{h}}(\operatorname{Ad}(g) \xi)\right)
$$

is constant for $g \in G$. Then $b\left(\operatorname{pr}_{\mathfrak{h} \cap \mathfrak{k}}(\operatorname{Ad}(g) \xi), \operatorname{pr}_{\mathfrak{h} \cap \mathfrak{k}}(\operatorname{Ad}(g) \xi)\right)$ is constant for $g \in K$. In other words $\xi$ defines a constant length Killing vector field on $K /(K \cap H)$.

If $\mathfrak{k} \cap \mathfrak{h}=\mathfrak{k}$ then $\mathfrak{k} \subset \mathfrak{h}$. The adjoint action of $\mathfrak{k}$ on $\mathfrak{g}$ is the sum of two copies of the adjoint representation of $\mathfrak{k}$, which is irreducible, so $\mathfrak{k} \subset \mathfrak{h}$ says that either $\mathfrak{h}=\mathfrak{k}$ or $\mathfrak{h}=\mathfrak{g}$. If $\mathfrak{h}=\mathfrak{k}$ there is no nonzero Killing vector field of constant length on $G / H$. If $\mathfrak{h}=\mathfrak{g}$ then $G / H$ is reduced to a point. So $\mathfrak{k} \cap \mathfrak{h} \neq \mathfrak{k}$.

If $\mathfrak{k} \cap \mathfrak{h}=0$ then $b(\mathfrak{k}, \mathfrak{h})=0$ so $\xi \in \mathfrak{k} \subset \mathfrak{m}$. Then $\operatorname{pr}_{\mathfrak{m}}(\xi)=\xi$ and $\operatorname{pr}_{\mathfrak{h}}(\xi)=0$. In particular, $b\left(\operatorname{pr}_{\mathfrak{m}}(\operatorname{Ad}(g) \xi), \operatorname{pr}_{\mathfrak{m}}(\operatorname{Ad}(g) \xi)\right)=b(\xi, \xi)$ and $b\left(\operatorname{pr}_{\mathfrak{h}}(\operatorname{Ad}(g) \xi), \operatorname{pr}_{\mathfrak{h}}(\operatorname{Ad}(g) \xi)\right)=0$ for all $g \in G$. Now $\operatorname{Ad}(G) \xi \subset \mathfrak{m}$, so $\mathfrak{m}$ contains a nonzero ideal of the simple Lie algebra $\mathfrak{g}$. In other words $\mathfrak{m}=\mathfrak{g}$ and $\mathfrak{h}=0$, so $M$ is the group manifold $G$.

Now suppose $\mathfrak{k} \cap \mathfrak{h} \neq 0$. As $\mathfrak{k} \cap \mathfrak{h} \varsubsetneqq \mathfrak{k}$ and $\xi$ defines a constant length Killing vector field on $K /(K \cap H)$, we know from [11] or from Theorem 4.4 that $(\mathfrak{k}, \mathfrak{k} \cap \mathfrak{h})$ is one of

$$
\text { (a) }(\mathfrak{s u}(2 n), \mathfrak{s p}(n)), \quad\left(\text { b) }(\mathfrak{s o}(2 n), \mathfrak{s o}(2 n-1)), \quad \text { or }(\mathrm{c})\left(\mathfrak{s o}(7), \mathfrak{g}_{2}\right) .\right.
$$

Here $(\mathfrak{h}, \mathfrak{k} \cap \mathfrak{h})$ is a symmetric pair, $\mathfrak{h} \cap \mathfrak{k}$ is simple by (5.4), and of course $\mathfrak{k} \neq \mathfrak{h} \subset \mathfrak{g}$.

Decompose $\mathfrak{h}=\mathfrak{h}_{1} \oplus \mathfrak{h}_{2}$ where $\mathfrak{h}_{2} \cap \mathfrak{k}=0$ and every ideal of $\mathfrak{h}_{1}$ has nonzero intersection with $\mathfrak{k}$. Then $\mathfrak{h}_{2} \subset \mathfrak{p}$ and $\mathfrak{h} \cap \mathfrak{k}=\mathfrak{h}_{1} \cap \mathfrak{k}$. Thus (5.4) limits the possibilities of $\mathfrak{h}_{1}$ to

$$
\begin{aligned}
&(5.4 \mathrm{a}): \mathfrak{h}_{1}=(\mathrm{i}) \mathfrak{s p}(n ; \mathbb{C}),(\mathrm{ii}) \mathfrak{s l}(n ; \mathbb{H}),(\text { iii }) \mathfrak{e}_{6, \mathfrak{c}_{4}}(n=4), \\
&(5.4 \mathrm{~b}): \mathfrak{h}_{1}=(\mathrm{iv}) \mathfrak{s o}(2 n-1 ; \mathbb{C}),(\mathrm{v}) \mathfrak{s l}(2 n-1 ; \mathbb{R}),(\text { vi }) \mathfrak{f}_{4 ; \mathfrak{b}_{4}}(n=5), \\
&(5.4 \mathrm{c}): \mathfrak{h}_{1}=(\text { vii }) \mathfrak{g}_{2, \mathbb{C}} .
\end{aligned}
$$

We eliminate case (iii) of (5.5) because $\mathfrak{e}_{6}$ has no nontrivial representation of degree 8 , and (vi) because $\mathfrak{f}_{4}$ has no nontrivial representation 
of degree 10. In case (v), passing to the complexification we would have $\mathfrak{s l}(2 n-1 ; \mathbb{C}) \subset \mathfrak{s o}(2 n ; \mathbb{C}) \oplus \mathfrak{s o}(2 n ; \mathbb{C})$ while $\mathfrak{s l}(2 n-1 ; \mathbb{C})$ has no nontrivial orthogonal representation of degree $2 n$; that eliminates case (v). At this point we notice that $\mathfrak{h}_{1}$ is a maximal subalgebra of $\mathfrak{g}$, so $\mathfrak{h}=\mathfrak{h}_{1}$.

Case (ii) is more delicate. The analog of $[\mathbf{1 1}]$ reduces the existence of a Killing vector field $\xi$ of constant length to the question of whether $\xi^{\prime}=i\left(\begin{array}{cc}-1 & 0 \\ 0 & 1\end{array}\right)$ defines a Killing vector field of constant length on $M^{\prime}=S L(2 ; \mathbb{C}) / S L(1 ; \mathbb{H})$. Since $M^{\prime}$ is the noncompact Riemannian symmetric space $S L(2 ; \mathbb{C}) / S U(2)$, the answer is negative. We have eliminated cases (ii), (iii), (v) and (vi) of (5.5), and we have shown $\mathfrak{h}=\mathfrak{h}_{1}$.

At this point we have shown that there is a nonzero elliptic $\xi \in \mathfrak{g}$ such that $\xi^{M}$ has constant length, if and only if $(G, H)$ is one of the four pairs listed in Theorem 5.2. If $(G, H)=(S L(2 n ; \mathbb{C}), S p(n ; \mathbb{C}))$ we can take $\xi=i \operatorname{diag}\{2 n-1 ; 1, \ldots, 1\}$; it is centralized by $G L(2 n ; \mathbb{C})$. If $(G, H)=$ $(S O(2 n ; \mathbb{C}), S O(2 n-1 ; \mathbb{C}))$ we can take $\xi=\operatorname{diag}\{J, \ldots, J\}$ where $J=$ $\left(\begin{array}{cc}0 & 1 \\ -1 & 0\end{array}\right)$; it is centralized by $G L(n ; \mathbb{C})$. If $(G, H)=\left(\operatorname{Spin}(7 ; \mathbb{C}),\left(G_{2}\right) \mathbb{C}\right)$ we can take $\xi$ in a Cartan subalgebra dual to a short root. As noted in Remark 5.3, if $(G, H)=(G,\{1\})$ we can take $\xi$ to be any element of the Lie algebra $\mathfrak{g}$ acting by right translations. Looking at the compact versions, in all cases one calculates $\operatorname{dim} Z_{G}(\xi) /\left(Z_{G}(\xi) \cap H\right)=\operatorname{dim} G / H$, so Lemma 5.1 ensures that the Killing vector field $\xi^{M}$ has constant length.

q.e.d.

Remark 5.6. Here is another argument to eliminate case (ii) of (5.5) in the proof of Theorem 5.2. $\mathfrak{g}_{\mathbb{C}} \cong \mathfrak{g} \oplus \overline{\mathfrak{g}}$ with $\mathfrak{g}$ embedded diagonally, so $\mathfrak{g}_{\mathbb{C}}$ has compact real form $\mathfrak{g}_{u} \cong \mathfrak{k} \oplus \mathfrak{k}$ with $\mathfrak{k}$ embedded diagonally. Now $\xi \in \mathfrak{k}$ has form $\xi=\left(\xi^{\prime}, \xi^{\prime}\right)$ inside $\mathfrak{g}_{\mathbb{C}}$, so it has nontrivial projections to each of the two simple summands of $\mathfrak{g}_{u}$. This is impossible here because $H_{u}$ is the diagonal $S U(2 n)$ inside $G_{u} \cong S U(2 n) \times S U(2 n)$.

\section{Summary for $G$ simple}

Combining Theorems 4.4 and 5.2 we arrive at

Theorem 6.1. Suppose that $G$ is simple. Then there is a nonzero elliptic element $\xi \in \mathfrak{g}$ such that the Killing vector field $\xi^{M}$ on the normal homogeneous space $M=G / H$ has constant length, if and only if, up to finite covering, $(G, H)$ is one of the following:

1. $(S U(2 p, 2 q), S p(p, q))$ with $p+q=n,(S L(2 n ; \mathbb{R}), S p(n ; \mathbb{R}))$ or $(S L(2 n ; \mathbb{C}), S p(n ; \mathbb{C}))$;

2. $(S O(2 p+2,2 q), S O(2 p+1,2 q))$ with $p+q=n$ or $(S O(2 n+2 ; \mathbb{C}), S p(2 n+1 ; \mathbb{C}))$;

3. $\left(\operatorname{Spin}(7), G_{2}\right),\left(\operatorname{Spin}(3,4),\left(G_{2}\right)_{\mathbb{R}}\right)$ or $\left(\operatorname{Spin}(7 ; \mathbb{C}), G_{2, \mathbb{C}}\right)$;

4. $(G,\{1\})$. 
Looking through this listing one sees

Corollary 6.2. Suppose that $G$ is simple, and that $\xi \in \mathfrak{g}$ is nonzero and elliptic. Let $L$ be the centralizer of $\xi$ in $G$. Then the following are equivalent:

1. $\xi^{M}$ has constant length on $M=G / H$.

2. L has an open orbit on $G / H$.

3. $H$ has an open orbit on the flag domain $G / L$.

\section{The three cases}

Retain the notation of Section 2. Note that $G_{u}$ acts transitively on the complex flag manifold $Z=G_{\mathbb{C}} / Q$, so $Z=G_{u} / L_{u}$ where $L_{u}$ is a compact real form of $L$. This expresses $Z$ as a compact simply connected homogeneous Kähler manifold.

By coset space reduction of $G / H$ we mean a decomposition $G=$ $G^{\prime} \times G^{\prime \prime}$ (locally) such that $H=\left(H \cap G^{\prime}\right) \times\left(H \cap G^{\prime \prime}\right)$, and, consequently, $G / H=\left(G^{\prime} /\left(H \cap G^{\prime}\right)\right) \times\left(G^{\prime \prime} /\left(H \cap G^{\prime \prime}\right)\right)$, with each factor of positive dimension. We will say that $G / H$ is coset space irreducible if there is no such nontrivial reduction. The following is immediate from the definitions:

Lemma 7.1. Suppose that $G$ is semisimple and $G / H=G^{\prime} / H^{\prime} \times$ $G^{\prime \prime} / H^{\prime \prime}$ is a coset space reduction. If $b$ is an invariant bilinear form on $\mathfrak{g}$ then $b=b^{\prime} \oplus b^{\prime \prime}$ where $b^{\prime}$ (resp. $b^{\prime \prime}$ ) is an invariant bilinear form on $\mathfrak{g}^{\prime}$ (resp. $\left.\mathfrak{g}^{\prime \prime}\right)$. The corresponding decomposition $\mathfrak{g}=\mathfrak{h}+\mathfrak{m}$ breaks up as $\mathfrak{g}^{\prime}=\mathfrak{h}^{\prime}+\mathfrak{m}^{\prime}$ and $\mathfrak{g}^{\prime \prime}=\mathfrak{h}^{\prime \prime}+\mathfrak{m}^{\prime \prime}$ where $\mathfrak{h}=\mathfrak{h}^{\prime} \oplus \mathfrak{h}^{\prime \prime}, \mathfrak{m}=\mathfrak{m}^{\prime} \oplus \mathfrak{m}^{\prime \prime}, \mathfrak{m}^{\prime}$ is the $b^{\prime}$ orthocomplement of $\mathfrak{h}^{\prime}$ in $\mathfrak{g}^{\prime}$, and $\mathfrak{m}^{\prime \prime}$ is the $b^{\prime \prime}$-orthocomplement of $\mathfrak{h}^{\prime \prime}$ in $\mathfrak{g}^{\prime \prime}$. In particular, the corresponding factors of the pseudo-Riemannian product decomposition are normal homogeneous spaces.

Let $M=G / H$ with $G$ reductive. Up to finite coverings we then have a decomposition

$$
G=G_{0} \times G_{1} \times \cdots \times G_{s} \times G_{s+1} \times \cdots \times G_{r},
$$

where $G_{0}$ is commutative, $G_{i}$ is simple for $i>0$. $H$ is the (isomorphic) image of a reductive Lie group $\widetilde{H}$ under a homomorphism $\varphi(x)=\left(\varphi_{0}(x), \ldots, \varphi_{r}(x)\right)$ where $\varphi: \widetilde{H} \rightarrow G_{i}$. We are going to study constant length Killing vector fields on $M=G / H$ defined by vectors

$$
\begin{aligned}
& \xi=\xi_{0}+\cdots+\xi_{r} \in \mathfrak{g}, \text { where } \\
& \qquad \xi_{i} \in \mathfrak{g}_{i}, \xi_{i} \neq 0 \text { for } 1 \leqq i \leqq s \text { and } \xi_{i}=0 \text { for } s<i \leqq r .
\end{aligned}
$$

In view of Lemma 7.1 we need only consider the coset irreducible cases. There are three basic possibilities of reductive normal coset irreducible $G / H$ : 
(i) for some index $i$ we have $\varphi_{i}(\widetilde{H})=\{1\}$,

(ii) for every index $i$ we have $\{1\} \neq \varphi_{i}(\widetilde{H}) \varsubsetneqq G_{i}$, and

(iii) for some index $i$ we have $\varphi_{i}(\widetilde{H})=G_{i}$.

The first of these cases is somewhat trivial:

Lemma 7.5. Let $M=G / H$ be coset space irreducible with some $\varphi_{i}(\widetilde{H})=\{1\}$ then $G=G_{i}=M$ and every $\xi \in \mathfrak{g}_{i}$ defines a constant length Killing vector field on $M$.

Proof. The hypothesis says that $G_{i}=G_{i} / \varphi_{i}(\widetilde{H})$ is a factor in a coset space reduction of $G / H$, and coset space irreducibility says that $G_{i}=$ $G_{i} / \varphi_{i}(\widetilde{H})$ must be all of $G / H$. As given, $G_{i}$ acts isometrically on itself by left translations, and by normality the right translations also are isometries. If $\xi \in \mathfrak{g}$ comes from the left action of $G$ on itself, it is centralized by the right action, which is transitive, so the corresponding vector field $\xi^{M}$ has constant length.

q.e.d.

Now we may (and do) assume that each $\operatorname{dim} \varphi_{i}(\widetilde{H})>0$. The second case is

Theorem 7.6. Assume that $M=G / H$ is a coset space irreducible normal homogeneous space with $G$ semisimple and $H$ reductive in $G$. In the notation of $(7.2)$ suppose that $\varphi_{i}(\widetilde{H}) \varsubsetneqq G_{i}$ and $\operatorname{dim} \varphi_{i}(\widetilde{H})>0$ for each $i>0$. Let $\xi=\xi_{0}+\cdots+\xi_{r} \in \mathfrak{g}$, elliptic and decomposed as in (7.3). Consider the following conditions:

(1) $\xi$ defines a constant length Killing vector field $\xi^{M}$ on $M=G / H$.

(2) For each $i, \xi_{i}$ defines a constant length Killing vector field $\xi_{i}^{M_{i}}$ on $M_{i}=G_{i} / \varphi_{i}(\widetilde{H})$.

(3) For each $i, \xi_{i}$ defines a constant length Killing vector field $\xi_{i}^{M}$ on $M$.

(4) The $\operatorname{Ad}(G)$-centralizer of $\xi$ has an open orbit on $M$. Then

(a) (1) implies (2) but (2) does not imply (1);

(b) (2) and (3) are equivalent; and

(c) (1) and (4) are equivalent.

Proof. As in the first paragraph of the proof of Theorem 5.2 we may assume that $\xi$ is contained in $\mathfrak{k}=\mathfrak{g} \cap \mathfrak{g}_{u}$.

We write $L$ and $L_{u}$ for the respective centralizers of $\xi$ in $G$ and $G_{u}$ and $Z$ for the complex flag manifold $Z=G_{u} / L_{u}=G_{\mathbb{C}} / Q$. We also write $L_{i}$ and $L_{i, u}$ for the respective centralizers of $\xi_{i}$ in $G_{i}$ and $G_{i, u}$, so $Z$ is the product of the $Z_{i}=G_{i, u} / L_{i}$, where of course $L_{0}=G_{0}$ and $L_{i}=G_{i}$ for $i>s$, so those $Z_{i}$ are single points. 
We first prove that (1) implies (2) and (4). As a subgroup of $G$, $H_{u}$ acts holomorphically and isometrically on $Z$. Here $Z$ carries the $G_{u}$-invariant Kaehler metric whose associated symplectic form is the Kostant-Souriau form. The action is Hamiltonian. We are assuming (1), in other words that $\xi^{M}$ has constant length on $M=G / H$, so Proposition 3.3 says that $\xi^{M_{u}}$ has constant length on $M_{u}=G_{u} / H_{u}$. In other words the momentum map for the action of $H_{u}$ on $Z$ has constant square norm. Thus [3, Theorem 1] $Z=Z^{\prime} \times Z^{\prime \prime}$, holomorphically and isometrically, where $Z^{\prime}$ and $Z^{\prime \prime}$ are complex flag manifolds such that $H_{u}$ is transitive on $Z^{\prime}$ and $H_{u}$ acts trivially on $Z^{\prime \prime}$.

The group $H_{u}$ acts nontrivially on $Z_{i}$ for $1 \leqq i \leqq s$. For if the action were trivial then $\varphi_{i}\left(\widetilde{H_{u}}\right)$ would be normal in $G_{i, u}$, while it is $\neq\{1\}$, forcing $\varphi_{i}\left(\widetilde{H_{u}}\right)=G_{i, u}$. This possibility was excluded by hypothesis. Thus $Z^{\prime}=Z_{1} \times \cdots \times Z_{s}$. Now set $G^{\prime}=G_{1} \times \cdots \times G_{s}, L^{\prime}=L_{1} \times \cdots \times L_{s}$, $\varphi^{\prime}=\varphi_{1} \times \cdots \times \varphi_{s}$ and $H^{\prime}=\varphi^{\prime}(\widetilde{H})$. Then $H_{u}^{\prime}$ is transitive on $Z^{\prime}$. It follows from [10, Proposition 2.1] that $H_{i, u}^{\prime}:=\varphi_{i}\left(\widetilde{H}_{u}\right)$ is transitive on $Z_{i}$ for $1 \leqq i \leqq s$. Equivalently $G_{u}^{\prime}=H_{u}^{\prime} L_{u}^{\prime}$, which is the same (take inverses) as $G_{u}^{\prime}=L_{u}^{\prime} H_{u}^{\prime}$, so $L_{u}^{\prime}$ is transitive on $Z^{\prime}$. In particular, $G_{u, i}=L_{u, i}^{\prime} H_{u, i}^{\prime}$. Thus $\xi_{i}^{M_{i, u}}$ has constant length on $M_{i, u}$ for $1 \leqq i \leqq s$. Thus (1) implies (2) and (4), and (4) implies (1) by Lemma 5.1.

It is obvious that (3) implies (2). Given (2), the centralizer $L_{i}$ of $\xi_{i}$ in $G_{i}$ is transitive on $M_{i}$, so the centralizer of $\xi_{i}$ in $G$ is transitive on $M$, and (3) follows.

It remains only to show that (2) does not imply (1). Consider the case $G=S O(2 n) \times S O(2 n)$ with $H=S O(2 n-1)$ embedded diagonally and $\xi=\operatorname{diag}\{J, \ldots, J\}$ where $J=\left(\begin{array}{cc}0 & 1 \\ -1 & 0\end{array}\right)$. Then $L=U(n) \times U(n)$ and $L \cap H=U(n-1)$ so $\operatorname{dim} G / H=2 n^{2}+n-1>2 n^{2}=\operatorname{dim} L$, so $L$ cannot have an open orbit on $G / H$ when $n>1$. On the other hand the projections of $\xi$ to the ideals of $\mathfrak{g}$ define constant length Killing vector fields $\xi_{i}^{M_{i}}$ on the $M_{i}=G_{i} / \varphi_{i}(\widetilde{H})$ because $L_{i}=U(n)$ is transitive on $M_{i}=G_{i} / \varphi_{i}(\widetilde{H})=S O(2 n) / S O(2 n-1)=S^{2 n-1}$. Thus $(2)$ does not imply (4). But (1) and (4) are equivalent, so (2) does not imply (1).

q.e.d.

The third case includes the pseudo-Riemannian group manifolds $(H \times H) /(\operatorname{diag}\{H\})$ for real simple Lie groups $H$, but the following example shows that this case is more of a combinatorial problem than a geometric or Lie theoretic problem:

Example 7.7. Let $G^{\prime}$ and $G^{\prime \prime}$ be reductive Lie groups. Let $\widetilde{H}$ be reductive with homomorphisms $\varphi^{\prime}: \widetilde{H} \rightarrow G^{\prime}$ and $\varphi^{\prime \prime}: \widetilde{H} \rightarrow G^{\prime \prime}$ such that $h \mapsto\left(\varphi^{\prime}(h), \varphi^{\prime \prime}(h)\right)$ is an isomorphism of $\widetilde{H}$ onto a reductive subgroup $H$ of $G:=G^{\prime} \times G^{\prime \prime}$. Let $M=G / H$ be the corresponding homogeneous space with any $G$-invariant pseudo-Riemannian metric. Suppose that 
$\xi \in \mathfrak{g}^{\prime}$ and that $\varphi^{\prime}(\widetilde{H})=G^{\prime}$. Then $G^{\prime \prime}$ centralizes $\xi$ and $G=H G^{\prime \prime}$, so the centralizer of $\xi$ in $G$ is transitive on $M$. Thus $\xi^{M}$ has constant length on $M$. The most familiar case of this is a compact group manifold $(H \times H) /(\operatorname{diag}\{H\})$.

\section{References}

[1] L. Biliotti, On the moment map on symplectic manifolds. Bull. Belg. Math. Soc. 16, 107-116, MR2498962, Zbl 1160.53373.

[2] G. Fels, A. T. Huckleberry \& J. A. Wolf, Cycle Spaces of Flag Domains: A Complex Geometric Viewpoint. Progress in Mathematics, vol. 245, Birkhäuser/Springer, Boston, 2005. MR2188135, Zbl 1084.22011.

[3] A. Gori \& F. Podestà, A note on the moment map on compact Kähler manifolds, Ann. Global Anal. Geom. 16 (2004), 315-318, MR2097621, Zbl 1084.53068.

[4] G. D. Mostow, Some new decomposition theorems for semi-simple groups, Memoirs Amer. Math. Soc. 14 (1955), 31-54, MR0069829, Zbl 0064.25901.

[5] A. L. Onishchik, Inclusion relations among transitive compact transformation groups. Trudy Moskov. Mat. Obšč. 11 (1962), 199-142, Zbl 0192.12601 (in Russian); Amer. Math. Soc. Transl. (2) 50 (1966), 5-58 (in English), Zbl 0207.33604.

[6] A. L. Onishchik, Topology of Transitive Transformation Groups, Johann Ambrosius Barth, Leipzig/Berlin/Heidelberg, 1994, MR1266842, Zbl 0796.57001.

[7] J. A. Wolf, Sur la classification des variétés Riemanniènnes homogènes à courbure constante. C. R. Acad. Sci. Paris 250 (1960), 3443-3445, MR0115146, Zbl 0112.36701.

[8] J. A. Wolf, Isotropic manifolds of indefinite metric. Comment. Math. Helv. 39 (1964), 21-64, MR0179744, Zbl 0125.39203.

[9] J. A. Wolf, The action of a real semisimple group on a complex flag manifold, I: Orbit structure and holomorphic arc components. Bull. Amer. Math. Soc. 75 (1969), 1121-1237, MR0251246, Zbl 0183.50901.

[10] J. A. Wolf, Real groups transitive on complex flag manifolds. Proc. Amer. Math. Soc. 129 (2001), 2483-2487, MR1823935, Zbl 0968.22006.

[11] M. Xu \& J. A. Wolf, Killing vector fields of constant length on Riemannian normal homogeneous spaces, Transformation Groups 21 (2016), 891-902. DOI:10.1007/s00031-016-9380-y.

Department of Mathematics UNIVERSITY OF CALIFORNIA Berkeley, CA 94720-3840, USA E-mail address: jawolf@math.berkeley.edu

Dipartimento di Matematica e Informatica "U.Dini"

Viale Morgagni 67/A I-50100-FirENZE, ITALY

E-mail address: podesta@math.unifi.it

College of Mathematics

TIANJIN NORMal UNIVERSITY

Tianjin 300387, P.R. China

E-mail address: mgmgmgxu@163.com 